www.jmscr.igmpublication.org

Index Copernicus Value: 79.54

ISSN (e)-2347-176x ISSN (p) 2455-0450

crossrefDOI: https://dx.doi.org/10.18535/jmscr/v7i1.170

\title{
Acute polyneuropathy resembling Guillain Barre Syndrome in Eosinophilic Granulomatosis with Polyangiitis
}

\author{
Authors \\ Dr Kamalasanan C.G ${ }^{1 *}$, Dr Lakshmy $M^{2}$, Dr Aquil Kalanad ${ }^{3}$, Dr Kiran Kamalasanan \\ ${ }^{1}$ Additional Professor, Department of Internal Medicine, Government Medical College, Calicut, Kerala, \\ India \\ ${ }^{2}$ Senior Resident, Department of Internal Medicine, Government Medical College, Calicut, Kerala, India \\ ${ }^{3}$ Assistant Professor, Department of Internal Medicine, Government Medical College, Calicut, Kerala, India \\ ${ }^{4}$ Senior Resident, Department of Surgical Oncology, Regional Cancer Centre, Thiruvananthapuram, Kerala,
} India

*Corresponding Author

Dr Kamalasanan. C.G

Additional Professor, Department of Internal Medicine, Government Medical College, Calicut, Kerala, India, 673008

Email:drkamal_315@hotmail.com,Mob:9847095038

\begin{abstract}
Eosinophilic granulomatosis with polyangiitis (EGPA) is an anti neutrophil cytoplasmic antibody related small vessel vasculitis which occurs exclusively in patients with asthma and eosinophilia. Vessel inflammation and eosinophilic infiltration leads to organ damage with high incidence of neurological involvement. We report a 58 year old patient who presented with rapidly ascending weakness with hyporeflexia mimicking Guillain Barre syndrome (GBS) clinically. A history of bronchial asthma, peripheral eosinophilia and eosinophilic infiltrates in sural nerve biopsy confirmed it to be a case of EGPA. He was treated with methylprednisolone and cyclophosphamide and had good response after completion of treatment. This case highlights the rare presentation of EGPA as GBS like polyneuropathy.

Keywords: Acute polyneuropathy, Guillain Barre Syndrome, Eosinophilic Granulomatosis with Polyangiitis, Churg Strauss syndrome.
\end{abstract}

\section{Introduction}

Eosinophilic granulomatosis with polyangiitis (EGPA) or Churg Strauss syndrome is a necrotising small vessel vasculitis associated with asthma and eosinophilia. It commonly presents with upper airway and lung involvement, peripheral neuropathy, cardiac involvement and skin lesions. Antineutrophil cytoplasmic antibodies (ANCA) is found to be positive in around $40 \%$ cases ${ }^{1}$. Peripheral nervous system involvement is seen in 50-75\% patients usually in the form of mononeuritis multiplex and symmetric peripheral neuropathy ${ }^{2}$.Wrist drop or foot drop is the cardinal manifestation of mononeuritis multiplex, and this is confirmed by nerve conduction studies or sural nerve biopsy. Diagnosis is made by demonstrating inflammation in vasa nervosum ${ }^{3}$.Guillain Barre syndrome(GBS) 
is a clinical syndrome of acute inflammatory polyneuropathy with features of ascending weakness, areflexia and minimal or no sensory loss. Two third of patients have symptoms of preceding respiratory or gastrointestinal tract infection within four weeks of onset of weakness ${ }^{4}$. The spectrum of the disease includes the main phenotypes acute inflammatory demyelinating neuropathy and acute motor axonal neuropathy ${ }^{5}$. We present a case of EGPA who had an initial clinical picture mimicking GBS. Early differentiation between two entities is essential as management strategies are different.

\section{Case Report}

A 58 year old male presented to us with complaints of sudden onset lower limb weakness followed by upper limb weakness along with severe pain. The weakness rapidly progressed in a symmetric fashion and increased in severity within 2 days. He had a history of upper respiratory tract infection 2 weeks before onset of complaint.

He giveshistory of bronchial asthma for 8 years and nasal polyposis for which he had undergone polypectomy. He had a history suggestive of transient ischemic attack. He denied history of smoking and alcoholism. $\mathrm{He}$ was taking bronchodilators and antiplatelets regularly. There was no history of intake of medications like antihistamines or leukotriene inhibitors.

On admission the patient was unable to walk. Neurological examination revealed normal higher functions. Cranial nerve function was normal. On motor examination there was severe motor weakness in predominantly in lower extremities (distal lower limbs, grade 0/5, proximal lower limb grade $2 / 5$, weak handgrip right hand, proximal upper limbs 5/5). Examination of sensory system showed sensory loss of all modalities of sensations from below midcalf. There was absent deep tendon reflexes with plantar reflex mute. Nothickened nerves. Respiratory system examination revealed rhonchi. Cardiovascular and alimentary system examination were normal. No skin lesions were present.

The initial examination was suggestive of acute polyneuropathy and a presumptive diagnosis of Guillain Barre syndrome was made in view of rapid progression, ascending weakness and hyporeflexia. He was planned to be treated with intravenous immunoglobulin while nerve conduction study was awaited. Nerve conduction study was of asymmetric sensorimotor axonal pattern which was strikingly different from the symmetric demyelinating pattern usually seen in GBS. CSF examination did not show any albumino-cytological dissociation. Laboratory study showed $\mathrm{Hb} 13.6 \mathrm{~g} / \mathrm{dl}$, marked peripheral eosinophilia (Total count: 22000, Differential Mixed: 53\%) which was confirmed by peripheral smear, ESR of $110 \mathrm{~mm}$ at $\mathrm{I} \mathrm{hr}$, normal liver and renal function tests.

A history of bronchial asthma, nasal polyposis, peripheral eosinophilia and axonal asymmetric polyneuropathy pointed towards a vasculitic complication of eosinophilic granulomatosis with polyangiitis or Churg Strauss syndrome.Right sural nerve biopsy showed dense inflammatory infiltrate composed of predominantly eosinophils with fibrinoid necrosis suggestive of vasculitic neuropathy (Figure 1,2). However, the patient was ANCA negative. CT paranasal sinuses (PNS) showed sino nasal polyposis (Figure 3).

Patient was treated with pulse methylprednisolone $1 \mathrm{~g}$ daily for three days, pulse cyclophosphamide $15 \mathrm{mg} / \mathrm{kg}$ every 2 weeks three doses followed by every 3 weeks six doses.After treatment his weakness and sensory symptoms gradually improved. The patient was able to walk with support upon completion of immunosuppressive treatment. 

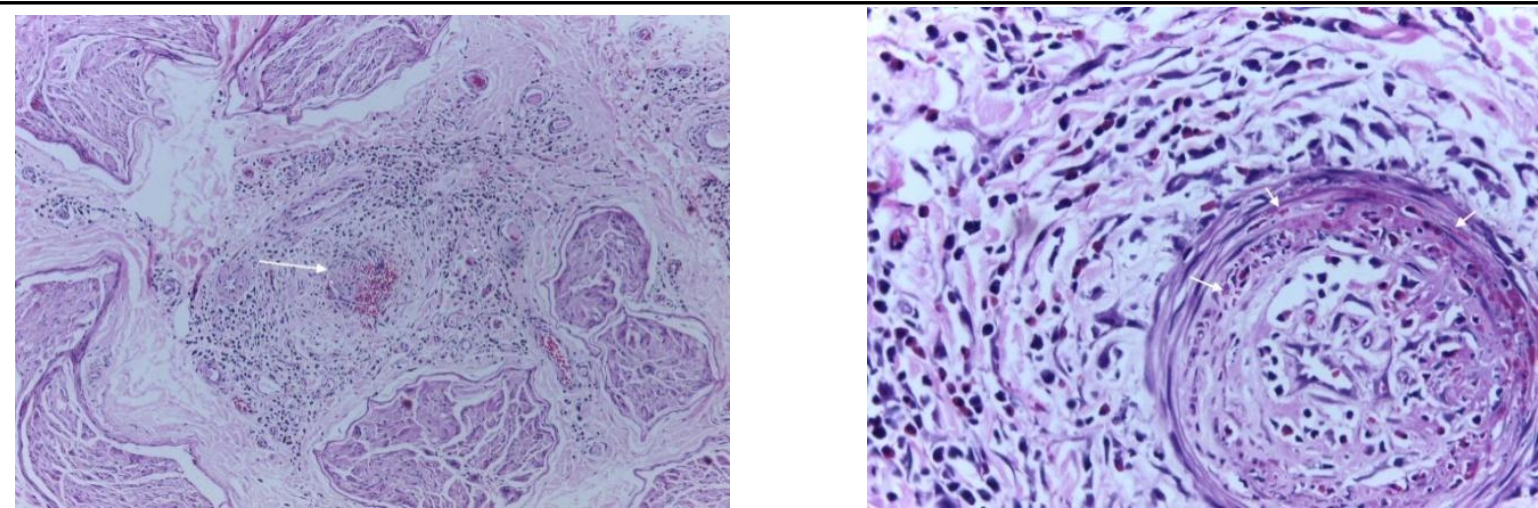

Figure 1,2: Sural nerve biopsy. Eosinophilic infiltrate around epineural vessel (left), fibrinoid necrosis and luminal thrombosis (right).

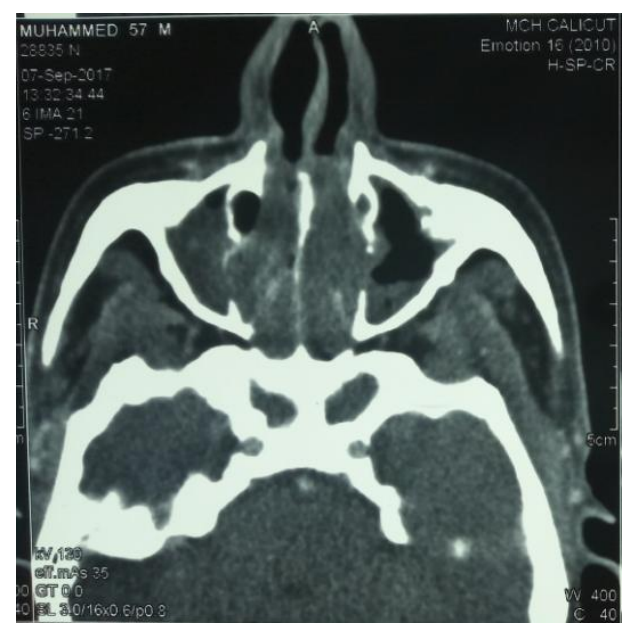

Figure 3: CT PNS showingsinonasal polyposis

\section{Discussion}

Herein we report a patient who presented with acute rapidly progressing polyneuropathy which made us to suspect GBS as the prima facie diagnosis.

EGPA is diagnosed in patients who has four out of the six criteria which are: 1.history of bronchial asthma 2.eosinophilia greater than $10 \%$ of total blood count 3. mononeuropathy or polyneuropathy 4. Migratory or transient pulmonary opacities detected radiographically 5. Paranasal sinus abnormality 6.biopsy containing blood vessel with extra vascular infiltration of eosinophils $^{6}$. Our patient had a history of bronchial asthma, paranasal sinus abnormality which made us to suspect EGPA as the underlying disease, leading to ascending weakness and hyporeflexia. The points which were against GBS was left upper limb sparing, significant sensory loss, axonal involvement in nerve conduction study and absence of albumino-cytological dissociation.
In a prospective study by $\mathrm{J}$. Wolf et $\mathrm{al}^{7}, 12$ out of 14 newly diagnosed EGPA patients had an involvement of peripheral nervous system. Mononeuritis multiplex was more common than acute polyneuropathy. Vasculitic neuropathy classically present as multiple mononeuritis but many overlapping mononeuritison different occasions can end up with a symmetric polyneuropathy $^{8}$. EGPA evolves through three stages, the prodromal stage characterised by bronchial asthma and rhino sinusitis, eosinophilic phase constitutes marked eosinophilia and organ involvement and a vasculitic phase with features of small vessel involvement. Peripheral neuropathy (PN)occurs in about $70 \%$ of patients, renal manifestations occur in $25 \%$ in the form of isolated urinary abnormalities to rapidly progressing glomerulonephritis and skin lesions such as palpable purpura and nodules predominates this phase of disease ${ }^{9,10}$.

Most important for timely treatment is early diagnosis which though difficult in prodromal 
phase should not be missed in vasculitic phase. Peripheral neuropathy in EGPA though is rarely life threatening, but can cause significant disability. In a study conducted by Hye-Jin Cho et $\mathrm{al}^{11}$ complete recovery of $\mathrm{PN}$ was observed in $66 \%$ of patients with sensory neuropathy and $83 \%$ of patients with limb weakness. Their patients received intravenous methylprednisolone pulse for three days followed by cyclophosphamide pulse monthly for six to eight months.

There are at least 8 case reports of EGPA mimicking GBS at presentation. Ngn et al reported the first case of EGPA which mimicked GBS clinically and electrophysiologically. Their patient had received plasmapheresis till his right wrist weakness worsened .The diagnosis was confirmed with persistent eosinophilia, positive ANCA and sural nerve biopsy ${ }^{12}$. Next case was reported in 1998 by Keven et al ${ }^{13}$ the patient presented with GBS like picture initially, later developed ANCA positive nephrotic syndrome.

Rapidity of progression of neuropathy, a history of bronchial asthma, peripheral eosinophilia and very high ESR made us to suspect a vasculitic process in our patient. It was confirmed by nerve conduction study and sural nerve biopsy. He was treated with intravenous methylprednisolone with cyclophosphamide which completely reversed his symptoms after 9 months of treatment. Prompt diagnosis could avert unnecessary treatment and financial burden of intravenous immunoglobulin with a probable diagnosisas GBS.

Hence, we conclude that EGPA can be a differential diagnosis of acute symmetric polyneuropathy, like GBS. A thorough history and investigations suggesting vasculiticaetiology mandates early initiation of immunosuppressive treatment which can lead to complete recovery.

\section{References}

1. Vaglio A, Buzio C, Zwerina J. Eosinophilic granulomatosis with polyangiitis (Churg Strauss):state of art.Allergy. 2013; 68:261-273.
2. Padoan R, Felicetti M, Marconato M, et al. FRI0387 Peripheral Neuropathy in Eosinophilic Granulomatosis with Polyangiitis (EGPA). Incat Disability Score to evaluate damage and to predict long term outcome in 50 Patients. Annals of the Rheumatic Diseases 2016;75:574575.

3. Lanham JG, Elkon KB, Pusey CD, Hughes GR. Systemic vasculitis with asthma and eosinophilia: A clinical approach to the Churg-Strauss syndrome. Medicine (Baltimore) 1984;63:65-81.

4. Jacobs $\mathrm{BC}(1)$, Rothbarth $\mathrm{PH}$, van der Meché FG et al. The spectrum of antecedent infections in Guillain-Barré syndrome: a case- control study. Neurology. 1998 Oct;51(4):1110-5.

5. Hugh J Willison, Bart C Jacobs, Pieter A van Doorn.Guillain Barre syndrome. Lancet 2016; 388: 717-27.

6. Yoo IH, Choi ST, Choi SH, Kim JM, Ahn SW. Eosinophilic Granulomatosis with Polyangiitis Presented as Acute Polyneuropathy and Cerebral Vasculitis. Exp Neurobiol. 2017;26(3):168-171.

7. J. Wolf, V. Schmitt, F. Palm, A. J. Grau, and R. Bergner, "Peripheral neuropathy as initial manifestation of primary systemic vasculitides," Journal of Neurology,2013. vol. 260, no. 4, pp. 1061-1070.

8. Lacomis D,Zivković SA.Approach to vasculitic neuropathies. Journal of Clinical Neuromuscular Disease. 2007.9(1):265276.

9. Keogh KA, Specks U. Churg-Strauss syndrome: clinical presentation, antineutrophil cytoplasmic antibodies, and leukotriene receptor antagonists.Am J Med. 2003 Sep;115(4):284-90.

10. Sinico R A,D Toma L.Renal involvement in Churg-Strauss syndrome. Am J Kidney Dis. 2006 May;47(5):770-9.

11. Cho HJ, Yune S, Seok JM, et al. Clinical Characteristics and Treatment Response of 
Peripheral Neuropathy in the Presence of Eosinophilic Granulomatosis with Polyangiitis (Churg-Strauss Syndrome): Experience at a Single Tertiary Center. J Clin Neurol. 2016;13(1):77-83.

12. KKP Ngn, HM Yeung. Acute fulminant neuropathy in a patient with Churg-Strauss syndrome. Post graduate medical journal.1997.vol 73,no 858,pp .236-238.

13. K. Keven, G. Nergisoglu, S. Erturk et al., "A case of Guillain-Barre syndrome complicated by nephrotic syndrome and pANCA positivity". Nephron.1998. vol. 80, no. 3, pp. 361-362. 